REGARDS

SUR LECONOMIE ALLEMANDE

BULLETIN ECONOMIQUE DU CRAC

\section{Regards sur l'économie allemande}

Bulletin économique du CIRAC

$85 \mid 2008$

Varia

\title{
Deutsche Post AG : un géant mondial de la logistique
}

\section{Caroline Guiot}

\section{(2) OpenEdition}

\section{Journals}

Édition électronique

URL : http://journals.openedition.org/rea/486

DOI : $10.4000 /$ rea.486

ISBN : 978-2-8218-0866-9

ISSN : 1965-0787

\section{Éditeur}

CIRAC

\section{Édition imprimée}

Date de publication : 1 mars 2008

Pagination : 21-26

ISSN : 1156-8992

\section{Référence électronique}

Caroline Guiot, « Deutsche Post AG : un géant mondial de la logistique », Regards sur l'économie allemande [En ligne], 85 I mars 2008, document 2, mis en ligne le 01 mars 2010, consulté le 19 avril 2019. URL : http://journals.openedition.org/rea/486 ; DOI : 10.4000/rea.486 


\section{Deutsche Post AG : un géant mondial de la logistique}

\section{Caroline Guiot}

\begin{abstract}
Ponctuellement pour l'ouverture totale des services postaux ce $1^{\text {er }}$ janvier 2008, Deutsche Post AG (DPAG) se présente en géant mondial de la logistique, avec un chiffre d'affaires consolidé de plus de 60,5 milliards $€$, dont $59 \%$ sont générés hors de RFA (2006). Le groupe continue d'investir massivement pour l'avenir, comme le prouvent toutes les annonces relayées par la presse en ce début 2008 : le 24 janvier, John Allan, directeur de la division Logistique du groupe, déclare qu'une lettre d'intention a été signée entre DPAG et HP pour la mise en œuvre prochaine du programme de réduction des coûts au sein du groupe, se traduisant par l'externalisation d'une partie de ses systèmes informatiques devant générer plus d'un milliard d'euros d'économies sur les sept prochaines années. Le 28 janvier, Charles Graham, directeur de DHL Aviation, filiale de DPAG dans le fret aérien, annonce, aux côtés du directeur de Lufthansa Cargo, le lancement en 2009 d'une compagnie aérienne commune sous forme de joint-venture : AéroLogic, basée à Leipzig .
\end{abstract}

Ce positionnement est le fruit d'une stratégie menée sur plus d'une décennie. Désétatisée en 1994, puis partiellement privatisée et introduite en bourse à partir de novembre 2000, l'ancienne administration s'est systématiquement préparée à la levée de son monopole. Si DPAG a eu à gérer l'épineuse question de la modification des statuts de son personnel, ménageant la transition entre fonction publique et droit privé, le groupe a aussi épuisé toutes les possibilités de mettre à profit la rente de monopole que lui offrait son statut transitoire, recourant à une pratique procédurière systématique et jusqu'à la tentative de faire bénéficier l'appellation "Post» de la protection d'une marque. Aujourd'hui, la hausse sensible des planchers salariaux (SMIC légal allant jusqu'à 9,80€ à l'ouest, voir dans ce numéro) introduite par l'action conjointe des partenaires sociaux et du gouvernement fédéral pour les salariés des concurrents de DPAG limite les 'risques' dans une branche intensive en main d'œuvre faiblement qualifiée et où la concurrence s'effectue essentiellement par la compétitivité-prix...

Deutsche Post World Net (DPWN) est le nom de communication employé par le groupe, traduisant plus que jamais le maillage du réseau construit à l'échelle mondiale pour assurer la gestion et le transport des lettres, marchandises et informations. L'entité juridique reste Deutsche Post AG, dont les actions sont cotées en bourse depuis novembre 2000. Si le gouvernement fédéral s'est formellement retiré du capital de l'ancienne société publique, l'Etat demeure indirectement présent au capital, via les $30,6 \%$ des parts détenues et gérées à titre transitoire par la banque publique $\mathrm{KfW}$. Si la privatisation totale est prévue par la loi, l'échéance n'est pas encore fixée. Ou pour le dire autrement : l'environnement boursier ne semble pas assez porteur pour que la mise sur le marché des parts publiques restantes permette au budget fédéral la 'prise de bénéfice' espérée. Le gouvernement fédéral est donc directement intéressé par la bonne marche des affaires (et une valorisation avantageuse) de son ex-administration.

De ce fait, une partie de la considérable croissance de DPAG, dont le chiffre d'affaires a presque triplé en dix ans, est due au statut fiscal particulier de l'opérateur, exonéré de la TVA (19\%). Ainsi que le rappelle la Commission des monopoles dans son rapport de décembre 2007 sur l'état de la concurrence sur le marché
Deutsche Post World Net : le nom d'un réseau mondial

DPAG jouit d'un privilège fiscal 
Ouverture totale du marché allemand de la lettre à la concurrence en 2008

postal allemand, DGAP est le seul opérateur historique de réseaux à bénéficier de ce privilège ; même Deutsche Telekom est soumise au régime commun de la TVA depuis 1999. L'entrave à la concurrence qui en découle a amené la Commission européenne à engager en 2007 auprès de la Cour européenne de Justice une procédure d'infraction contre l'Allemagne, en cours de délibération.

En tant qu'entreprise partiellement privatisée dans un marché en voie de libéralisation, DPAG œuvrait dans un segment totalement ouvert à la concurrence depuis longtemps (le courrier express et la logistique) et dans un autre, celui qui s'est ouvert au marché le 1er janvier 2008 : la lettre, où le monopole de DPAG (« licence exclusive ») a été maintenu entre 1998 et 2008, bien que le périmètre en ait été progressivement réduit à $50 \mathrm{~g}$. Dans la catégorie des envois d'un poids supérieur à ce plafond, mais inférieur à $1000 \mathrm{~g}$, DPAG affronte depuis 1998 la concurrence de sociétés soumises au régime des licences, attribuées par l'autorité de régulation Bundesnetzagentur. Quant à la quatrième activité de DPAG : les services financiers, elle se situe dans une zone de relatif flou juridique entre le public et le privé. Si l'ouverture du marché domestique depuis le 1er janvier 2008 puis, entre 2009 et 2013, du marché européen de la lettre constitue un challenge pour sa situation sur le marché allemand, DPAG compte la saisir comme une chance de conquérir les autres marchés postaux européens.

Deutsche Post AG : chiffres clés 2006

\begin{tabular}{|c|c|c|c|c|c|}
\hline Divisions & $\begin{array}{l}\text { Chiffre d'affaires } \\
\quad(e n \text { mio } €)\end{array}$ & $\begin{array}{l}\text { Poids de la } \\
\text { division dans } \\
\text { le CA total }{ }^{\star}\end{array}$ & $\begin{array}{c}\text { Résultat } \\
\text { opérationnel } \\
\text { (en mio } €)\end{array}$ & $\begin{array}{l}\text { Poids de la } \\
\text { division dans } \\
\text { le RO total (*) }\end{array}$ & $\begin{array}{l}\text { Nombre de } \\
\text { salariés }^{\star *} \text { ) }\end{array}$ \\
\hline Lettre & 13286 & $19,9 \%$ & 2054 & $52,6 \%$ & 129922 \\
\hline Express & 17195 & $25,7 \%$ & 325 & $8,3 \%$ & 124280 \\
\hline Logistique & 22739 & $34,0 \%$ & 762 & $19,5 \%$ & 162706 \\
\hline Services financiers & 9593 & $14,3 \%$ & 1004 & $25,7 \%$ & 22570 \\
\hline Services & 4048 & $6,1 \%$ & -237 & $-6,1 \%$ & 23872 \\
\hline Consolidation & -6316 & - & -36 & - & - \\
\hline Deutsche Post AG (DPWN) & 60545 & $100,0 \%$ & 3872 & $100,0 \%$ & 463350 \\
\hline
\end{tabular}

Source des données : Deutsche Post World Net, Geschäftsbericht 2006. ( $\left.{ }^{*}\right)$ Somme des chiffres d'affaires et résultats opérationnels des divisions, hors Consolidation ; ${ }^{* *}$ équivalent temps plein, au 31 décembre 2006.

Lettre : la principale contribution au résultat opérationnel

Un réseau de distribution en cours de rationalisation

Le marketing direct et la distribution de presse défendent leurs parts de marché
Certes, sous l'effet de la concurrence émergente notamment, le poids du chiffre d'affaires de la division Lettre dans le CA total du groupe ne cesse de s'effriter, passant de 45,8\% en 1999 à moins de $20 \%$ en 2006. Mais cette activité reste le principal contributeur en termes de résultat opérationnel, pesant plus de $52,6 \%$ en 2006. L'activité Lettre, dirigée par Hans-Dieter Petram, est composée aujourd'hui de plusieurs segments : le courrier de communication représente en 2006 un volume de 9335 millions de plis acheminés, soit une légère baisse de $3 \%$ par rapport à 2005, qui s'explique aisément par la concurrence de la communication électronique qui s'étend. Héritage du monopole, la part de marché de Deutsche Post AG est encore aujourd'hui d'environ $90 \%$ sur ce segment. Restent les philatélistes (plus d'un million). La nouveauté 2008 pour eux et pour tous les particuliers autant que pour les entreprises, réside dans la possibilité de personnaliser les enveloppes comme les timbres, grâce à des photos ou des logos. Cette initiative vient à point nommé pour tenter de contrer les premières collections de timbres éditées par le concurrent Pin Group.

La problématique de la distribution du courrier est un point essentiel pour la division Lettre. DPAG communique relativement peu sur ses points de vente : environ 12000 guichets. Mais l'exigence de rentabilité et l'obligation de service universel ont amené le groupe à d'une part sous-traiter certaines activités (voir dans ce numéro) et d'autre part à filialiser un certain nombre de ses bureaux : c'est ainsi que 850 centres ont été transférés juridiquement à Postbank, la filiale des services financiers de DPAG.

Le second segment regroupe les solutions aux professionnels: le marketing direct, dont le volume accuse, pour la première fois en 2006, un léger repli. L'offre de DPAG est étendue : du conseil jusqu'au suivi des effets d'une campagne, en 
passant par la conception, la réalisation et la diffusion des documents sur tout type de support (papier, téléphone, mail). Grâce à l'éventail des solutions proposées et à son savoir-faire, Deutsche Post AG se prévaut d'une part de marché d'environ 13,5\% (selon ses propres estimations). Le marché de la distribution de presse enregistre un recul de $1 \%$ par rapport à 2005, à 18,2 milliards d'envois, qui s'explique par la diminution du nombre d'abonnés, mais aussi par l'extension du réseau de distribution propre aux éditeurs de presse en prévision de l'ouverture totale du marché. Parallèlement, Deutsche Post AG observe une augmentation du poids des envois et donc proportionnellement du prix moyen. Dans ce contexte, la part de marché de Deutsche Post AG est stable, autour de 11,4\%, selon ses propres estimations.

Pour compléter cette offre aux professionnels, le groupe veut prendre position sur le marché des services à valeur ajoutée. Grâce à l'acquisition de la société Williams Lea, basée à Londres et spécialisée dans le traitement des documents et données d'entreprises, le groupe propose les compétences d'un leader dans la gestion des dossiers : de la numérisation ou de l'impression à l'archivage en passant par le tri et les transferts de données papier ou numériques.

La gamme de prestations proposées ne serait pas complète sans l'acheminement des courriers internationaux, marché sur lequel le groupe reste leader, malgré l'ouverture à la concurrence depuis 2003, avec des connections directes dans plus de 200 pays, une part de marché estimée à $14 \%$, et des concurrents tels que UPS (pour une part de marché équivalente), Royal Mail (9\%), La Poste (8\%), SPI (6\%), Spring/TNT (5\%), et autres acteurs (44\%). Par ailleurs, la frontière entre les différentes activités du groupe est ténue: depuis le 1er janvier 2007 , les envois de paquets nationaux en Allemagne sont gérés au sein de la division Lettre et non plus Express comme auparavant. Ici, les principaux concurrents sont DPD, UPS, Hermes et GLS.
Elargissement de l'offre de gestion complète des documents

Paquets nationaux et courriers internationaux

\begin{tabular}{|lrr||lrr|}
\hline \multicolumn{1}{|c}{ Lettre } & \multicolumn{1}{c||}{} & \multicolumn{3}{c|}{ Logistique } \\
CA par segment & mio€ & \multicolumn{1}{c|}{$\%$} & CA par segment & mio $€$ & $\%$ \\
Courrier de communication & 6147 & $46 \%$ & & & \\
Marketing direct & 2766 & $21 \%$ & DHL Global Forwarding & 9271 & $39 \%$ \\
Distribution de presse & 818 & $6 \%$ & DHL Freight & 1999 & $8 \%$ \\
Courrier international et service à valeur ajoutée & 2818 & $21 \%$ & DHL Exel Supply Chain & 11957 & $51 \%$ \\
Autre & 737 & $6 \%$ & Consolidation & 488 & $2 \%$ \\
Lettre & $\mathbf{1 3 2 8 6}$ & $100 \%$ & Logistique & $\mathbf{2 2 ~ 7 3 9}$ & $100 \%$ \\
\hline
\end{tabular}

Source des données : Deutsche Post World Net, Geschäftsbericht 2006.

Sous la marque DHL, l'activité Express dessert quatre zones géographiques (Europe, Amériques, Asie-Pacifique et Pays émergents) avec une triple offre: « same-day », « time-definite », « day-definite ». Les perspectives de croissance de cette activité se jouent sur la qualité du service client autant que sur les capacités de Deutsche Post $A G$ à construire un réseau planétaire, reliant les régions et les clients, et en profitant de la croissance mondiale.

\begin{tabular}{|lrl|lrr|}
\hline \multicolumn{7}{c|}{ CA de l'activité Express par zone géographique } \\
Europe & 10106 & $54 \%$ & Pays émergents & 969 & $5 \%$ \\
Amériques & 4379 & $24 \%$ & Consolidation & 702 & $4 \%$ \\
Asie Pacifique & 2443 & $13 \%$ & Total Express & $\mathbf{1 7 1 9 5}$ & $\mathbf{1 0 0} \%$ \\
\hline
\end{tabular}

Source des données : Deutsche Post World Net, Geschäftsbericht 2006.

Et si DHL Express domine très largement les marchés sur trois des quatre zones géographiques (Europe, Asie-Pacifique et Pays émergents), la concurrence reste vive sur la zone Amériques. Pourtant, la présence sur ce continent est un axe stratégique pour DPAG, car il représente le plus important marché des courriers express. De plus, l'activité est générée autant par les échanges sur l'espace intérieur américain que par les liens avec le reste du monde qui tirent la croissance de ce marché. Par ailleurs, partout dans le monde, le développement important des
DHL Express leader en Europe, Asie-Pacifique et Pays émergents 
DHL Logistique, numéro un mondial du fret intercontinental

L'automatisation, gage de rentabilité et de qualité

La pénurie de main d'œuvre qualifiée

Deutsche Postbank le réseau de guichets le plus dense d'Allemagne ventes en ligne (e-business) et par correspondance n'est pas sans effet sur l'activité express, facteur d'accroissement des volumes gérés sur ce marché. C'est dans ce contexte qu'a été annoncée la création d'une joint-venture entre DHL Express et Lufthansa Cargo lançant une compagnie aérienne de fret commune, basée sur l'aéroport de Leipzig qui a récemment vu étendre ses capacités de logistique.

Générant $34 \%$ du CA du groupe, la branche Logistique, dirigée par John Murray Allan, s'enorgueillit d'être numéro un mondial du fret intercontinental, par voie aérienne ou maritime, avec des parts de marché respectives de $11,6 \%$ et 8,6\% des volumes de fret mondiaux. A côté de DHL Global Forwarding qui assure le transport et la livraison de toute marchandise dans le monde, DHL Exel Supply Chain supporte l'activité liée aux contrats de logistique, c'est-à-dire les clients externalisant tout ou partie de leurs transports de marchandises, du producteur au grossiste ou au consommateur. Grâce à l'acquisition de la société Exel en 2005, DHL est leader sur ce segment, offrant un savoir-faire et une expertise de plusieurs années, dans des domaines très différents de production industrielle, de l'automobile à l'industrie pharmaceutique. Mais avec une part de marché de $5 \%$ seulement sur le marché américain, DHL est encore déficitaire (1 milliard $€$ selon Die Welt, 19-02-08) et pourrait être contraint de s'adosser à un autre intégrateur (le nom d'UPS est évoqué). Enfin, sous la dénomination DHL Freight, c'est l'activité de fret ferroviaire et routier qui a été lancée en juillet 2006, née de la demande des clients et de la synergie existant au sein de DHL avec les deux métiers précités.

Les prestations de logistique de DHL s'étendent du perfectionnement des différents types de packaging au suivi satellite des colis et marchandises. Les investissements importants et l'automatisation croissante de l'ensemble des procédés tout au long de la chaîne logistique, de l'étiquetage au tri, en passant par l'acheminement et le suivi individualisé concourent d'une part à la rationalisation des coûts et donc à la rentabilité, et d'autre part à la qualité reconnue des prestations. Récompensée à plusieurs reprises en 2006 pour l'ensemble des services proposés et pour la qualité des prestations autant que des relations clients, la branche Logistique tire le groupe dans le sillage de sa dynamique positive.

Compte tenu de la forte croissance du marché, il n'est pourtant pas étonnant que soit constatée dans un dossier spécial Logistique publié par le quotidien Frankfurter Allgemeine Zeitung (21-01-08), la pénurie généralisée de main d'œuvre qualifiée dans le secteur. Bien que les 50 écoles et universités allemandes spécialisées forment 3000 logisticiens par an, Frank Straube, président de la Fédération nationale de la logistique (Bundesvereinigung Logistik, $B V L$ ), remarque que le marché du travail offre 5000 postes pour jeunes diplômés par an, tous métiers confondus. Car lorsque la flotte allemande s'accroit de 1000 bateaux en 6 ans, ce sont autant d'ingénieurs qu'il faudrait pouvoir embaucher. De même pour le fret aérien... La globalisation et l'extrême modernisation des activités ont profondément modifié les métiers de la logistique. C'est pourquoi la main d'œuvre qualifiée est recherchée, y compris aux échelons de qualification de technicien. DPAG en forme peu en comparaison de ses concurrents qui ont commencé à offrir des formations conformes aux nouveaux besoins de la branche. Ils ont ainsi défini deux nouveaux métiers (avec les formations en alternance afférentes), agréés en 2005 par la Fédération allemande des chambres d'industrie et de commerce : technicien et technicien commercial (Fachkraft et Kauffrau/mann) des services courrier, express et poste.

La branche Services financiers du groupe Deutsche Post AG est dirigée par Wulf von Schimmelmann, et assurée sous la marque Postbank, elle-même organisée en quatre pôles : Retail banking (banque de détail : services d'épargne, de prêts et d'assurance à destination des particuliers), Corporate banking (banque d'entreprise : investissements, financements, leasing), Transaction banking, et marchés financiers. Depuis janvier 1999, date à laquelle Postbank a rejoint le groupe DPAG (après en avoir brièvement été séparée), l'entreprise poursuit l'élargissement de son offre, grâce à plusieurs rapprochements stratégiques, à commencer par l'acquisition de DSL Bank, spécialisée dans les prêts et produits financiers 
complexes, en juin 1999. En juillet 2001, l'acquisition de BHF Holding a été un tremplin permettant à Postbank de se positionner sur le marché des financements internationaux, en particulier aux Etats-Unis. En janvier 2006, grâce à l'acquisition, approuvée par les autorités anticartels, de BHW Holding, banque créée en 1928 et spécialisée dans l'épargne, les financements immobiliers et les prêts hypothécaires, Postbank complète sa gamme de services aux particuliers et aux entreprises. Parallèlement, au début de l'année 2006, ce sont 850 bureaux de Deutsche Post AG qui ont été transférés sous forme de filiales et intégrés à la division des services financiers, créant le réseau de guichets le plus dense du pays, en comparaison de toute autre banque en Allemagne. C'est ainsi qu'en septembre 2006, le titre Postbank est intégré à l'indice du DAX - deux ans seulement après son introduction en bourse qui fut un grand succès populaire.

Dans ces conditions, Postbank (15 millions de clients) devient particulièrement attractive, et les rumeurs d'une fusion avec une grande banque privée enflent dans la presse. Un rachat par Commerzbank ou Deutsche Bank, intéressées par le projet, donnerait naissance à la plus grande banque de détail allemande, dont le réseau national serait à même de concurrencer celui des Caisses d'épargne (Sparkassen). L'information n'est certes pas officiellement commentée par DPAG, mais le ministre fédéral des Finances semble plaider en faveur d'une cession à Commerzbank. La volonté du gouvernement fédéral de séparer les activités postales et financières est sous-tendue par la même analyse que celle des actionnaires institutionnels de DPAG, dont DWS Investment : une vente 'par appartements' des dernières parts publiques dans DPAG semble largement plus rentable que la mise en bourse du conglomérat actuel. Le dossier pourrait aboutir rapidement, le Bund désirant refinancer le soutien qu'il vient d'accorder à l'IKB Industriebank (filiale de la KfW), mise à mal par la crise des subprimes.

Sur le chapitre social, DPAG se contente de mener une communication très 'politiquement correcte'. Si les perspectives d'emploi sont exposées clairement en termes d'activités à temps plein et à temps partiel, la ventilation de ces modalités de travail, pas plus que la répartition par type de contrat (à durée déterminée, indéterminée ou l'utilisation des services d'intérimaires), ni la gestion des anciens contrats de fonctionnaires hérités d'avant la privatisation de DPAG, ne sont rendues publiques. Seule une étude de l'institut WIK a permis d'apporter quelques précisions en 2007 sur les conditions de travail (voir dans ce numéro), et une estimation de la part des salariés soumis à un régime spécial du fait de la privatisation partielle (la moitié environ). Ces conditions, et notamment les salaires versés, se situent au dessus des normes en usage chez les prestataires concurrents et même d'autres secteurs comparables.

La communication sur les sujets sociaux s'attache plutôt à la mise en valeur du bien-être des salariés, exposant les programmes destinés à l'équilibre entre vie professionnelle et vie privée, passant justement par l'annualisation du temps de travail et la valorisation des temps partiels... Outre la formation des futurs jeunes diplômés et des cadres à haut potentiel, futurs dirigeants internationaux du groupe, les programmes de formation visent explicitement la santé et surtout l'amélioration de la sécurité des salariés travaillant pour l'essentiel sur des voies de circulation (rues, aéroports, etc.). De telles actions de sensibilisation permettent à DPAG d'afficher des résultats d'arrêts de travail en baisse continue sur plusieurs années.

Le groupe DPAG est beaucoup plus prolixe sur la communication des actions de développement durable touchant tant l'environnement que les Hommes, servant ainsi une forte demande dans l'opinion: anniversaire du partenariat avec I'UNICEF, collaboration avec le WWF pour protéger la forêt en Indonésie, etc.

LE DEVELOPPEMENT EN DIX ANS DU GROUPE PARTIELLEMENT PRIVATISE Deutsche Post AG est saisissant : l'opérateur a pris le parti de l'ouverture programmée des marchés postaux à la concurrence pour s'offrir des perspectives de conquêtes et donc de croissance. En faisant siennes les lois du marché, d'acquisition en
Commerzbank et Deutsche Bank candidates au rachat de Postbank

Peu d'informations sur les composantes sociales du groupe

Un focus sur la sécurité des salariés

Des actions sociales tournées vers l'extérieur 
acquisition, l'ancienne administration publique s'est forgée un réseau mondial inégalé dans les domaines de l'acheminement de plis et le transport de marchandises. II est vrai que le groupe a mis à profit pour cela la rente de monopole que représentait son statut de détenteur de la «licence légale exclusive » dans le segment de la lettre et qu'il ne s'est pas privé par ailleurs de mener une action procédurière systématique pour écarter la concurrence, surtout en ce qui concerne les services à valeur ajoutée - les seuls offrant une réelle perspective de développement. De même, l'opérateur a contribué, de connivence avec le syndicat des services ver.di, à ériger de nouvelles barrières à ses concurrents dans le segment de la lettre en imposant via le législateur l'entrée en vigueur, le jour où tombait son monopole légal, d'un SMIC (de 8 à $9,80 € / h$ ) proche des salaires planchers (le plus bas est de $9,63 € / h$ ) appliqués en interne. En accroissant ainsi les charges salariales de ses concurrents, il réduit la marge leur permettant de proposer leurs prestations à des tarifs inférieurs aux siens - eux aussi fixés par l'autorité de régulation à un niveau anormalement élevé qui ne se justifie qu'en partie par les charges spécifiques liées à la privatisation de cette exadministration, comme le rappelle la Commission des Monopoles. De même, la poursuite de son exonération de la TVA au-delà de l'ouverture totale du marché postal contribue elle aussi à proroger le monopole de fait de DPAG.

Un coup de théâtre a entraîné le 18 février un changement de direction à la tête de groupe. Klaus Zumwinkel, dont le mandat expirait normalement fin 2008, a dû présenter sa démission au directoire suite à des soupçons de fraudes fiscales personnelles. Le président du conseil de surveillance, Jürgen Weber, a entériné cette décision et félicité au passage $\mathrm{K}$. Zumwinkel pour son action menée au cours des 18 années passées à la tête de DPAG. En "homme de réseaux " dans le monde politique et syndical, il avait accompagné la désétatisation puis la privatisation partielle de DPAG, et était l'un des principaux orchestrateurs de la prorogation du monopole de DPAG par tous les moyens. Suite aux derniers événements, le conseil de surveillance, avec l'accord et le soutien du Bund, a désigné Frank Appel à la direction du groupe.

Sera-t-il l'homme d'une nouvelle ère pour DPAG ? Acteur majeur en 2000 de l'intégration des sociétés DHL et Danzas au sein du groupe, ainsi que de l'introduction en bourse, il était le directeur de la division Logistique, procédant aux restructurations internes prévues et prolongeant le transfert des activités courrier international dans la division Logistique par le biais de plusieurs programmes d'amélioration et de réduction des coûts qui ont permis d'accroître la rentabilité. Son parcours est donc plus tourné vers l'adaptation à des marchés très concurrentiels que vers la conservation d'un monopole menacé. Cette ligne de conduite sera-t-elle poursuivie à la tête du groupe ? L'un des premiers dossiers qu'il aura à gérer le révélera: la convention tarifaire «maison » conclue dans la Division Lettre expire le 30 avril. Les syndicats, ver.di en tête, revendiquent une hausse de $6 \%$ des salaires. Le management, quant à lui, prône une hausse de la durée hebdomadaire du travail pour les 60000 préposés qui ont encore le statut de fonctionnaires : actuellement de $38,5 \mathrm{~h}$, elle devrait atteindre les $41 \mathrm{~h}$ désormais réglementaires dans la fonction publique allemande. En ce printemps expire également un pacte interne interdisant tout licenciement économique...

\section{Indications bibliographiques}

BOURGEOIS I., "La libéralisation du marché postal allemand, entre service public et marché ", Regards sur l'Economie Allemande, $\mathrm{n}^{\circ} 47 / 00$ et "La difficile émergence d'un marché de la lettre en RFA », Regards sur l'Economie Allemande, n65/04

BOURGEOIS I., "Transport routier et logistique en Allemagne : nouvelles configurations sectorielles ", in BOURGEOIS I. (dir), Allemagne 2001 - Regards sur une économie en mutation, Editions du CIRAC, 2001

www.dpwn.de ; www.deutschepost.de ; www.dhl.de ; www.postbank.de ; www.pin-group.net www.faz.net ; www.handelsblatt.com ; www.ftd.de 